\title{
Effects of age, word frequency, and noise on the time course of spoken word recognition
}

\author{
Kristin J. Van Engen ${ }^{1}$, Avanti Dey ${ }^{1,2}$, Nichole Runge ${ }^{1}$, Brent Spehar ${ }^{3}$ \\ Mitchell S. Sommers ${ }^{1}$, Jonathan E. Peelle ${ }^{3}$ \\ ${ }^{1}$ Department of Psychological and Brain Sciences, Washington University in St. Louis \\ ${ }^{2}$ Center for Vital Longevity, University of Texas at Dallas \\ ${ }^{3}$ Department of Otolaryngology, Washington University in St. Louis
}

Running title: Age, word frequency, and word recognition

Please address correspondence to:

Dr. Kristin Van Engen

Department of Psychological and Brain Sciences

Washington University in St. Louis

1 Brookings Drive

St. Louis, MO 63110

email: kvanengen@wustl.edu 


\begin{abstract}
This study assessed the effects of age, word frequency, and noise on the time course of lexical activation during spoken word recognition. Participants (41 young adults and 39 older adults) performed a visual world word recognition task while we monitored their gaze position. On each trial, four phonologically-unrelated pictures appeared on the screen. A target word was presented following a carrier phrase ("Click on the ___ "), at which point participants were instructed to use the mouse to click on the picture that corresponded to the target word. Highand low-frequency words were presented in quiet to half of the participants and in noise to the other half at a signal-to-noise ratio $(\mathrm{SNR})$ of $+3 \mathrm{~dB}$. Results show that, even in the absence of phonological competitors in the visual array, high-frequency words were fixated more quickly than low-frequency words by both listener groups. Young adults were generally faster to fixate on targets compared to older adults, but the pattern of interactions among noise, word frequency, and listener age show that older adults' lexical activation largely matches that of young adults in a modest amount of noise.
\end{abstract}




\section{Introduction}

Contemporary models of spoken word recognition generally agree on a lexical competition framework in which similar-sounding words (phonological neighbors) compete with each other for recognition, so that recognition difficulty is related to the number of competitors a word has in the lexicon (Luce \& Pisoni, 1998; Marslen-Wilson \& Tyler, 1980; Norris \& McQueen, 2008). Lexical representations are activated by incoming acoustic information, and listeners must select a response from the possible candidates while inhibiting incorrect options. A word like "cat", for example, has a large number of similar-sounding competitors (“cap", "calf", "hat"...) and would thus be more difficult to accurately perceive than a word like "orange", which has few competitors. A key characteristic of lexical competition frameworks is that these processes of lexical activation, inhibition, and selection operate even when recognition is successful (although errors also reflect word perception difficulty). While lexical activation may be partly automatic, selection and inhibition have been proposed to rely on additional cognitive resources (Mitchell S. Sommers \& Danielson, 1999). Thus, lexical competition may underlie at least a portion of the cognitive challenge associated with effortful listening (Peelle, 2018;

Pichora-Fuller et al., 2016).

The problem of effortful listening is particularly relevant for older adults, who experience increased difficulty with spoken word recognition, especially in the context of background noise. This difficulty is likely due to a combination of auditory and cognitive factors (Humes et al., 2013): in addition to high rates of hearing loss among older adults, which can limit auditory access to speech signals, there is evidence to suggest that they are particularly affected by the cognitive challenge associated with lexical competition. Sommers and Danielson (1999), for example, showed that older adults had disproportionate difficulty recognizing words with many phonological neighbors (i.e., from dense phonological neighborhoods) relative to young adults 
when they were tested at noise levels that equated the groups' recognition of words with few neighbors. In the current study, we examine the effect of another lexical factor-word frequency — on young and older adult listeners in both quiet and noisy conditions.

One productive approach to measuring lexical activation in the absence of recognition errors has been the visual world paradigm (Allopenna et al., 1998). In a typical visual world experiment, an array of pictures is presented on a screen in front of a participant, who hears a word and is asked to indicate what they heard. The direction of the participant's gaze is tracked and used to index lexical activation. Conveniently, then, eyetracking can be used to measure the activation of both target words and distractors in the visual array. For example, for the target word "beaker", competitors like "beetle" and "speaker" also receive some looks from listeners (Allopenna et al., 1998).

Using this paradigm, Dahan et al. (2001) investigated the effect of word frequency on word recognition in young adults. In their first experiment, phonologically-related words were presented together in visual arrays, and the ones that were higher in frequency were looked at by listeners more quickly than the lower-frequency competitors. In their second experiment, each target word (which was either high or low frequency) was presented with three phonologically unrelated words. Listeners looked to high frequency words more quickly than low frequency words, even in the absence of phonologically similar competitors. In the current study, we aimed to replicate this result and use it to investigate the effects of aging and noisy listening environments on the temporal dynamics of spoken word recognition.

We consider two broad sets of processes that contribute to correct word identification. First, auditory processes deal with the accumulation of sensory evidence for a particular word. At the beginning of a word, not enough information has been processed to correctly identify it; when the word is complete, the maximum amount of sensory evidence is available. However, 
differentiating acoustic characteristics and contextual information usually allow listeners to tell what word has been presented before the end of the word (Grosjean, 1980; Tyler \& Wessels, 1983; Wingfield et al., 1991). We expect that degrading the acoustic signal-for example, through the addition of background noise—will generally slow this process. Complementing auditory processes are cognitive factors that are important for both inhibiting responses that were initially activated but are no longer consistent with the acoustic input, and for selecting the correct word. Thus, two listeners with access to identical amounts of acoustic input may differ in spoken word recognition ability due to individual differences in their ability to select the appropriate word from among the possible competitors. Because of age-related changes in both hearing and cognition (e.g., general slowing), we expect older adults to show slower word recognition than young adults.

In visual word processing, word frequency tends to have a stronger influence on older adult readers compared to younger readers (Balota et al., 2004; Spieler \& Balota, 2000). To determine whether this pattern also characterized spoken word recognition, Revill and Spieler (2012) used the visual world paradigm, using visual arrays that included high- and lowfrequency targets as well as high- and low-frequency competitors. Their results showed that older adults were more likely than young adults to fixate on high-frequency competitors. However, the same study showed only a marginal effect of frequency on target recognition, leaving open the question of how word frequency affects target activation for older adults. Importantly, degrading the signal for young adult listeners in Revill and Spieler (2012) did not produce a similar competitor frequency effect, suggesting that the source of the frequency effect for older listeners is not hearing loss, but rather changes in the cognitive processes associated with word recognition. 
In the current study we examined spoken word recognition by young and older adults in the absence of phonological competitors among the visually-presented foils in order to focus on the speed of target activation. In this paradigm, a greater reliance on frequency in word recognition for older adults would predict a significant interaction between age and frequency, such that word frequency has a larger effect on the dynamics of word recognition for older than young adults.

\section{Materials and Methods}

Stimuli are available at https://osf.io/5kuct/.

\section{Materials}

Two hundred words were used for the experiment: 25 low-frequency targets (Log Freq HAL range of 5.1-6.8); 25 high-frequency targets (Log Freq HAL range of 10.0-11.9); and 150 midfrequency distractors (Log Freq HAL 6.9-9.3). All words were closed monosyllables that referred to imageable nouns. For each word, a color image on a white background was found online $(200 \times 200$ pixels $)$. Three distractor words were pseudo-randomly grouped with each of the critical words ensuring that none of the distractors were phonological neighbors or semantically related to the target. Distractors sharing the same onset as the critical word were also avoided. Fifty experimental displays were created out of these groups, with each of the four pictures in a different quadrant of the computer screen. The location of the target in each trial was randomized once and that location was used for all participants. The order of the trials was also randomized once, with this same order used for all participants.

Each display occurred with the spoken instructions "Click on the ". Recordings

were made by an American male from the Midwest. A single $1000 \mathrm{~ms}$ recording was used for the carrier phase, and recordings of each target word were appended to it. Half of the participants 
heard the stimuli in quiet, while the other half heard them in steady speech-shaped noise (created using the long-term average spectrum of the target word stimuli) at a signal-to-noise ratio (SNR) of $+3 \mathrm{~dB}$.

\section{Participants}

Participants were 41 young adults aged $18-25$ years $(25$ female, $M=21.2, S D=1.8)$ and 39 older adults aged $65-84$ years (24 female, $M=71.7, S D=5.1)$. Three additional young adults and 3 additional older adults participated, but their data were excluded because of problems with eyetracking (e.g., the eyetracker could not locate their eye) or because they fell asleep (one older participant). Given that this was the first eye-tracking study conducted by our research group, we did not have pilot or previous data on which to justify the sample size for this experiment. We therefore selected a sample size that was larger than those used in similar eyetracking studies (Revill and Spieler (2012) had 16 per age group; Dahan et al. (2001) had 18 in their group). Young adult participants were recruited from the undergraduate psychology pool at Washington University in St. Louis and received course credit or \$10/hour for their participation. Older adults were recruited from the St. Louis community. All participants were native English speakers, had self-reported normal or corrected-to-normal vision, did not use hearing aids, and were not color blind. All older adults scored at least 25 on the Mini Mental State Examination $(M=29.0, S D=$ 1.6) and had an average of 15.8 years of education $(S D=2.4)$. Young adults had a mean of 14.9 years of education $(S D=1.4)$ and were not administered the Mini Mental State Examination.

All participants were tested on vocabulary knowledge and hearing acuity. Vocabulary knowledge was assessed using the vocabulary subtest of the Wechsler Adult Intelligence Scale (Wechsler, 2008). To determine hearing acuity, pure-tone air-conduction thresholds were determined at 250, 500, 1000, 2000, 4000, and $8000 \mathrm{~Hz}$. A pure-tone average (PTA) was calculated for each listener in each ear by averaging the thresholds at 500, 1000, and $2000 \mathrm{~Hz}$. 
Group data are provided in Figure 1.
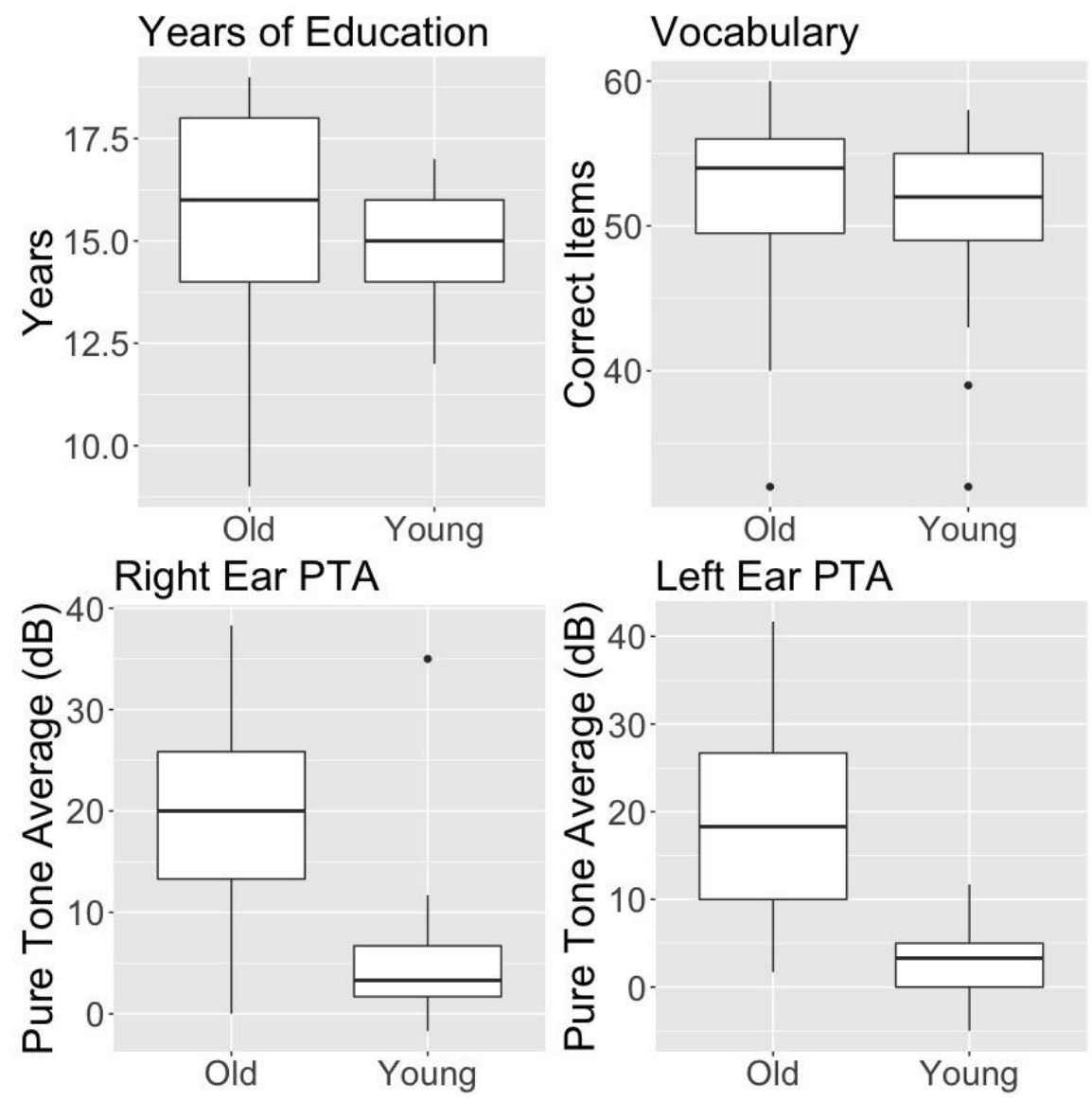

Figure 1. Education, vocabulary, and hearing information for young and older adult participants.

Unpaired t-tests showed statistically significant differences between the groups for hearing acuity in both ears (Left: $t=8.93, d f=47.84, p<.001$; Right: $t=8.64, d f=64.82, p<$ $.001)$. Their difference in years of education was marginal $(t=1.93, d f=60.77, p=0.06)$, and there was no significant difference in vocabulary $(t=0.98, d f=75.62, p=0.33)$.

\section{Procedure}

Participants were tested individually in a sound-attenuated booth. They were instructed that a fixation cross would appear in the center of the computer screen at the beginning of every trial. When ready, they should click on the cross. Upon clicking the cross, an experimental array with 
a picture in each of the four corners would appear on the screen and the phase "Click on the [TARGET]" would be heard through the speakers at a comfortable level. Using a mouse, the participant was instructed to move the cursor to the appropriate picture and click. A fixation cross would then appear, which they clicked to begin the next trial. Eye movements were tracked with a Tobii X120 eyetracker controlled by LabView 6.2 at a rate of 60 samples per second. Participants sat 0.5 meters from the screen, and a nine-point calibration procedure was conducted before testing began. Auditory stimuli were presented through a calibrated Madsen Auricle audiometer using two loudspeakers each approximately 1 meter from the listener and oriented +/-45 degrees from the participants' forward-looking position when facing the monitor.

\section{Results}

Data and analysis scripts are available from https://osf.io/5kuct/. Looks to the target were analyzed for the 1-second time window from $300 \mathrm{~ms}$ to $1300 \mathrm{~ms}$ after target word onset, and only trials in which the target was correctly recognized were included (accuracy was $>98 \%$ in all conditions). Each frame in the eyetracker output was simply coded as " 1 " if the eye was directed at the corner containing the target and " 0 " if it was not (i.e., frames where the eye was directed elsewhere or where the individual was blinking would be coded as 0 ).

We used logistic growth curve analysis (GCA) to model the by-participant target fixation data using the lme4 package in R. GCA is similar to polynomial regression, but controls for collinearity problems by orthogonalizing the polynomial time terms (Mirman, 2014). We modelled the time course with a third-order (cubic) orthogonal polynomial, and fixed effects were included for age (young vs. older), lexical frequency (high vs. low), and noise (quiet vs. noisy), along with the interactions among these three factors. The model also included 
participant and participant-by-frequency random effects to capture both overall individual differences and differences in the effect of the frequency manipulation on each subject. Statistical significance was determined using p-values based on asymptotic Wald tests (the default in the glmer function from the lme4 package in R). The full fixed effects output is included in the Appendix. Although all of the abovementioned factors were included in the model and in our considerations of statistical significance, we have plotted subsets of the effects to more clearly illustrate our results.
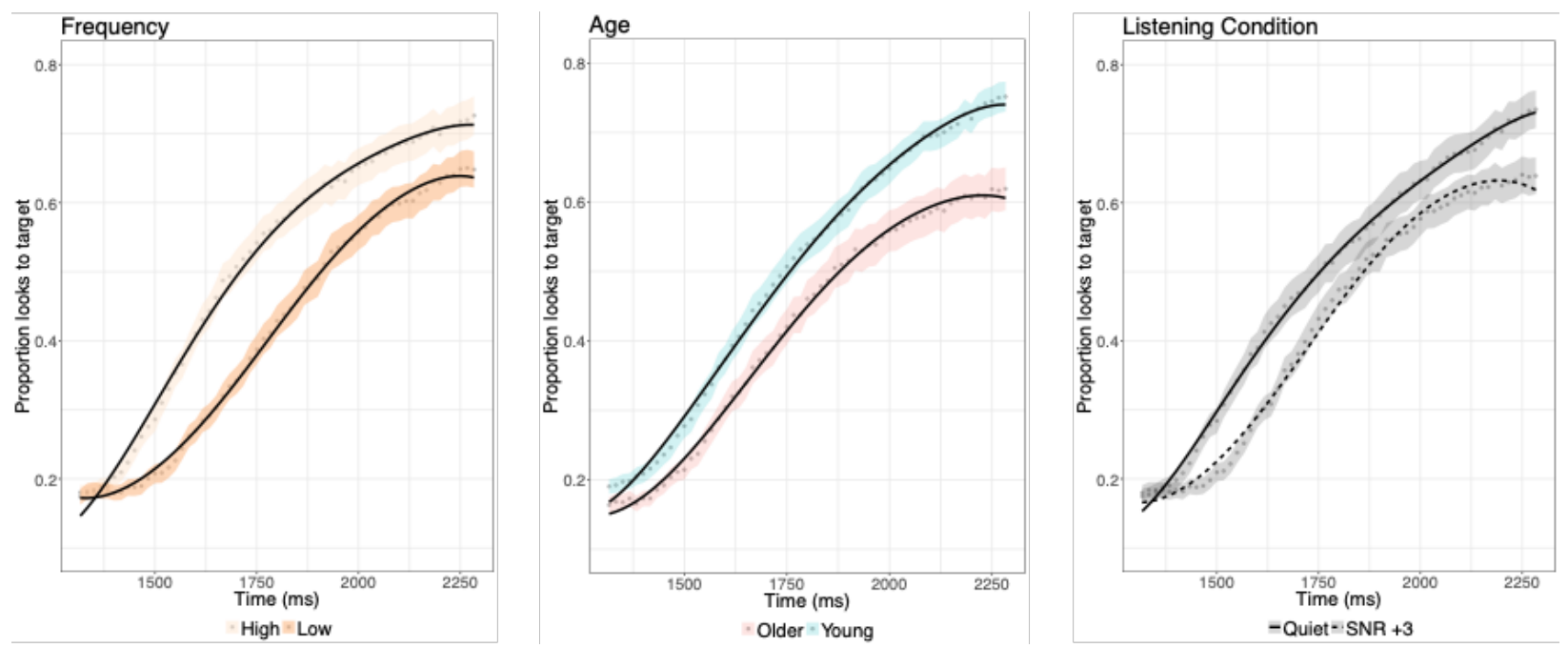

Figure 2. Fixed effects of word frequency, age, and noise (listening condition). Lines represent statistical model fits; dots represent raw averages; ribbons indicate standard error. One second of data is presented, beginning at $300 \mathrm{~ms}$ after target onset. Target onset occurred at $1000 \mathrm{~ms}$.

Figure 2 shows the simple effects of age, noise, and word frequency. Visual inspection of the data suggests there were fewer fixations to the targets overall for older adults vs. young adults, for noisy vs. quiet stimuli, and for low frequency words vs. high frequency words. The results of the growth curve analysis indicate that age was indeed a significant overall predictor ( $\beta$ $=-.30, S E=.16, z=1.93, p=.05)$. Age also significantly affected the linear time term $(\beta=-.60, S E$ $=.26, z=-2.26, p=.02)$, such that young adults' fixations to the target increased more rapidly than 
older adults' (see also Revill and Spieler, 2012). Noise significantly affected the cubic time term only $(\beta=.42, S E=.10, z=4.25, p<.001)$, as can be seen in the much more curved shape model for noisy presentations. High frequency words were fixated more than low frequency words overall $(\beta=.26, S E=.03, z=10.13, p<.001)$, and frequency interacted with all three time terms (linear: $\beta$ $=.36, S E=.15, z=2.45, p=.01 ;$ quadratic: $\beta=-.60, S E=.11, z=-5.46, p<.001 ;$ cubic: $\beta=.39, S E=$ $.08, z=4.87, p<.001)$.

There were also interactions between these factors, shown in Figure 3. Age interacted significantly with listening condition on the quadratic time term $(\beta=-.48, S E=.15, z=-3.26, p=$ $.001)$ and with frequency on the cubic term $(\beta=-.17, S E=.089, z=-2.10, p=.04)$. That is, the effects of noise and frequency differed for younger and older adults.
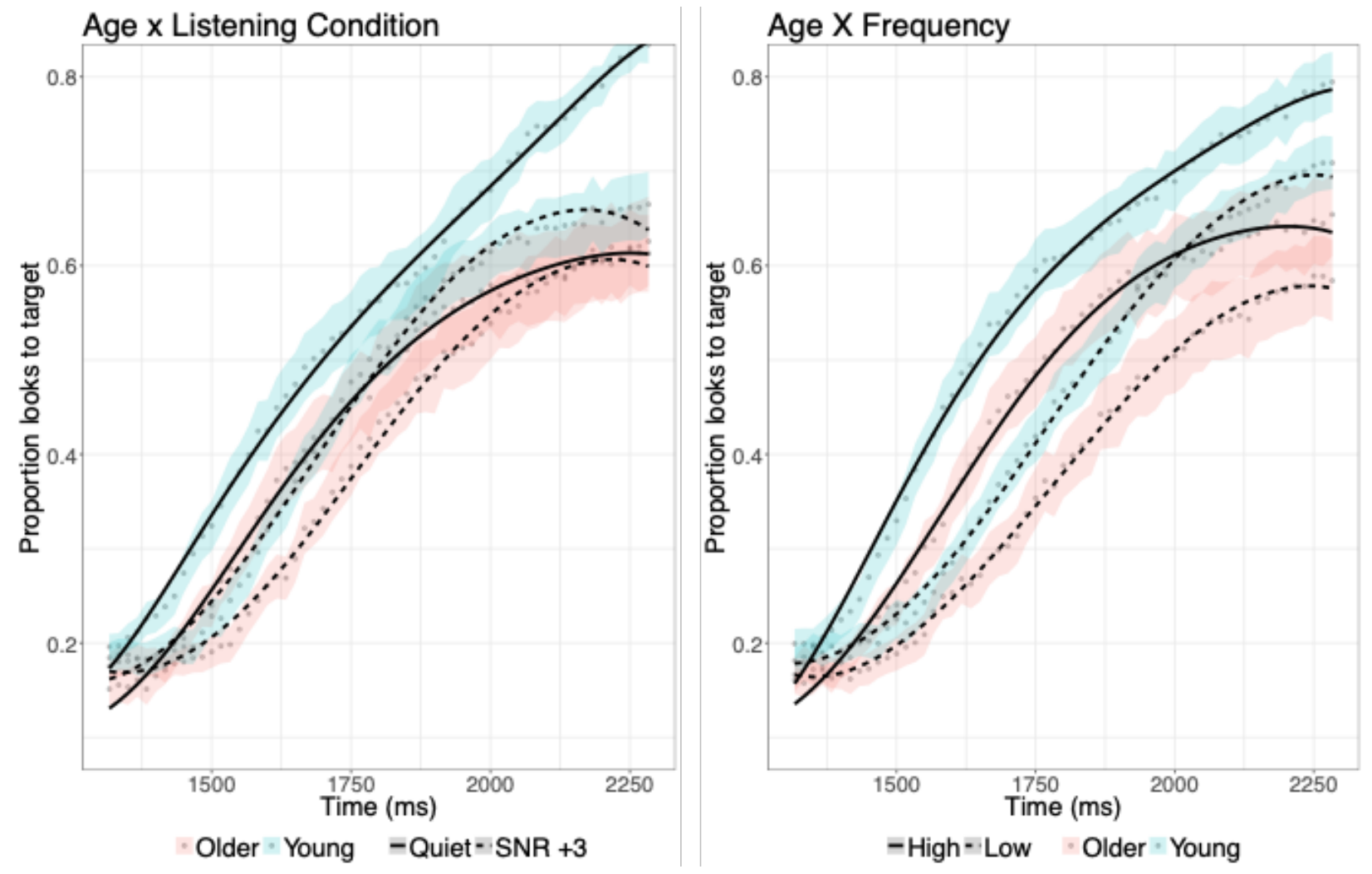

Figure 3. Significant two-way interactions between age and listening condition and between age and word frequency. Lines represent model fits; dots represent raw averages; ribbons indicate standard error. 
Inspection of the data shows that the interaction between age and listening condition arises because there is a larger effect of noise on the young adults: the model of the young adults' looks to the target words continues to increase in quiet but flatten in noise while the model of the older adults' fixations flattened in both listening conditions. The interaction between age and frequency on the cubic term similarly arises because the young listeners' modelled fixations to high-frequency targets continue to increase while their looks to low-frequency targets flatten like the older adults'. In both cases, then, older adults' looking behavior across conditions looks more similar to young adults' behavior in challenging conditions (low frequency words, noisy environment).

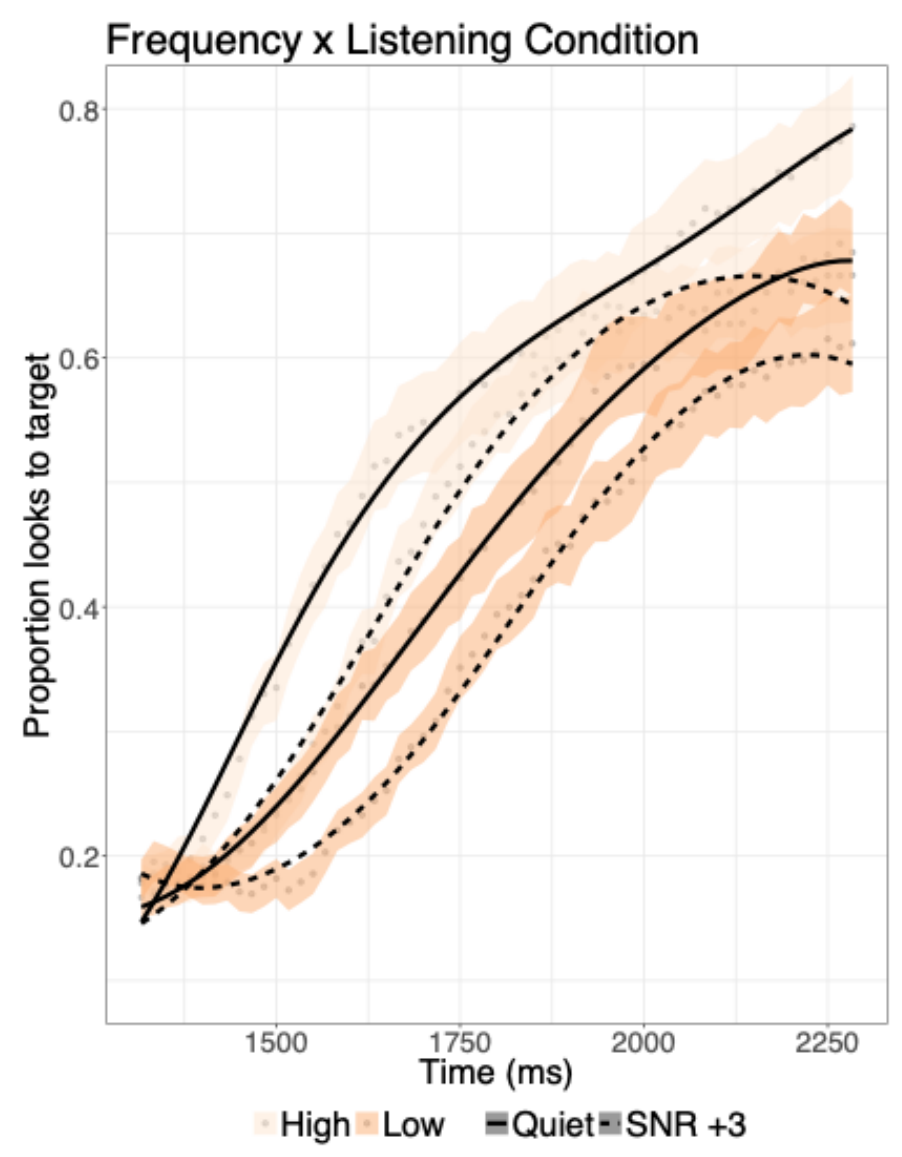

Figure 4. Significant interaction between word frequency and noise. Lines represent model fits; dots represent raw averages; ribbons indicate standard error. 
Word frequency and noise also interacted with one another on the quadratic time term $(\beta=.34$, $S E=.11, z=3.12, p<.01$ ). Visual inspection of this interaction (see Figure 4) shows that the model fit for high-frequency words in quiet is shaped quite differently from the other conditions, such that looks to the target were still increasing in the analysis time window for that condition only. Because of this, the noise effect for high-frequency words appears stronger than for lowfrequency words. It is also worth noting here that high frequency words in noise were recognized more quickly than low frequency words in quiet (i.e., the high-frequency data is all "above" the low-frequency data, even in noise.)

Finally, there was a three-way interaction among age, noise, and frequency on the linear time term $(\beta=-.31, S E=.15, z=-2.09, p=.04)$. This interaction likely arises because although only age and word frequency affect the linear time term in general, younger and older adults differ more from one another in quiet than in noise.

Hearing acuity was not included in the general analysis because of its correlation with age (Cruickshanks et al., 1998; Homans et al., 2017). A follow-up analysis restricted to the older adults was conducted with better-ear PTA as a fixed factor to assess whether hearing acuity would predict the time course of lexical activation. Despite the variability in hearing acuity among the older adults, there was no significant effect of better-ear PTA on the temporal dynamics of word recognition (i.e., inclusion of this variable did not improve the fit of the statistical model for older adults' gaze behavior).

\section{Comparison of young adults in noise to older adults in quiet}

Visual inspection of the data and the interactions above suggest that young adults in noise perform similarly to older adults in quiet. To test this statistically, we conducted a secondary analysis in which we compared young adults in noise to older adults in quiet (and removed the 
noise condition from the analysis). In this model, age, frequency, and their interaction were included as fixed effects, along with the same random effects structure as in the initial analysis. Only young adult performance in noise and older adult performance in quiet were included, so a significant effect of age would indicate that young adults in noise do not behave like older adults in quiet. The data and model fits are shown in Figure 5, and the full regression output can be found in Supplemental Materials.

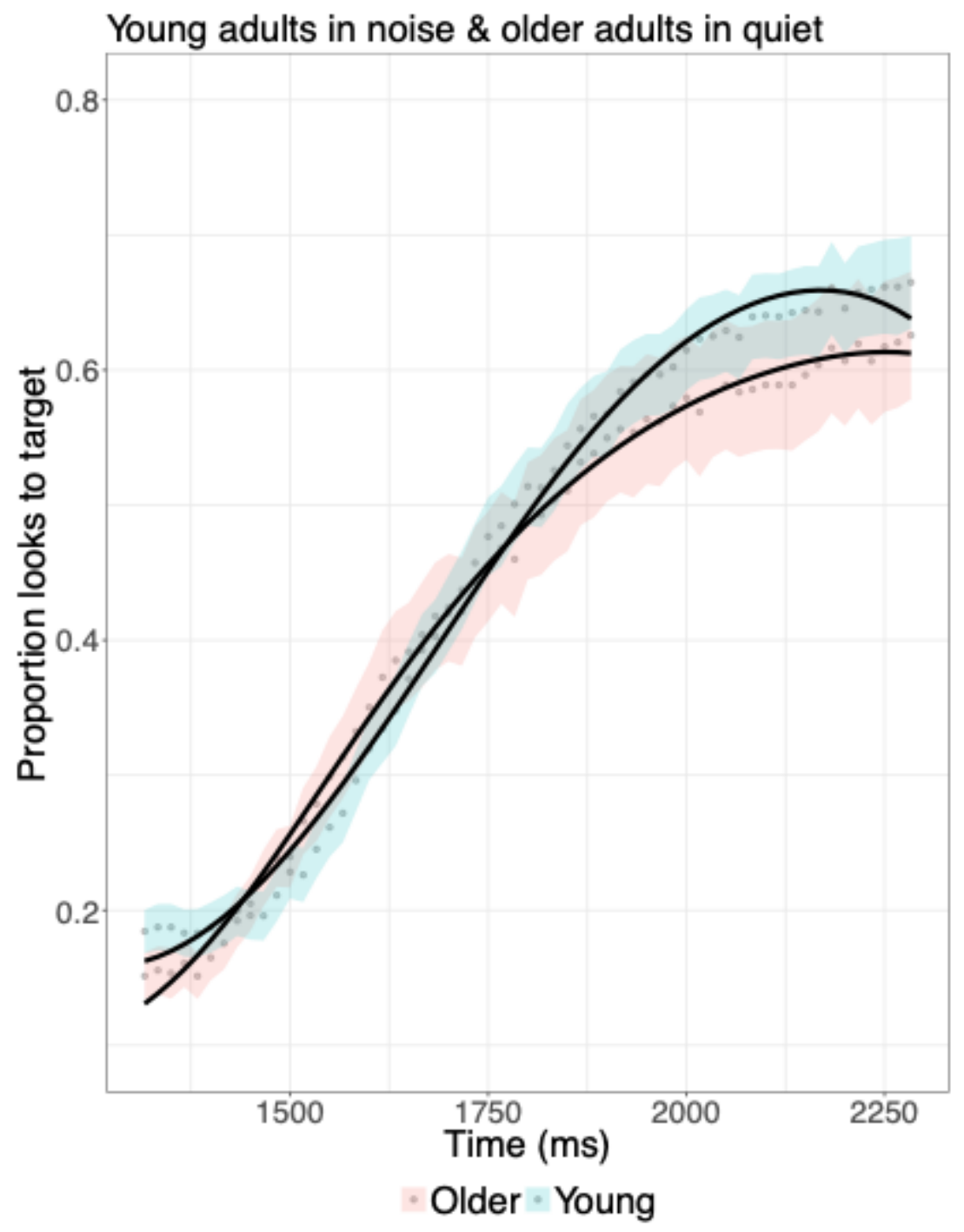

Figure 5. Young adults in $+3 \mathrm{~dB}$ SNR (green) and older adults in quiet (red).

As before, frequency had a significant effect overall $(\beta=.23, S E=.04, z=6.15, p<.001)$ and on all time terms in the model, but there was no overall effect of age $(\beta=-.22, S E=.30, z=-$ 
$.73, p=.46)$. The only significant result for age was on the cubic polynomial $(\beta=.31, S E=.16, z=$ $1.95, p=.05$ ). Visual inspection of the data suggests that this is because the fit to the young adult data has a sharper peak. The timing and rate of increase for the two groups, however, are very similar. Thus, the time course of young adult word recognition in an SNR of $+3 \mathrm{~dB}$ is largely similar to that of the older adults' in quiet. Furthermore, there was no significant interaction between age and frequency overall or on any of the time terms.

\section{Discussion}

It has long been observed that common words are recognized more rapidly and accurately than less common words (Goldinger et al., 1989; Howes, 1957; Marslen-Wilson, 1987). The current study replicated this lexical frequency effect in both young and older adult listeners using eyetracking with a visual array that did not include phonological competitors. Like Dahan et al. (2001), the current data show a very early influence of lexical frequency on the word-recognition process.

While the frequency effect was present in both age groups, we also observed differences between the age groups. First of all, younger adults generally looked to images depicting target words more quickly than older adults. While Revill and Spieler (2012) found that allowing age to affect the linear coefficient improved the fit of their growth curve model for target fixations and Ben-David et al (2011) found that older adults were slower than younger adults when they had to distinguish target words from rhyming alternatives, the current study is, to our knowledge, the first to show age-related slowing in the time course of word recognition using visual arrays that do not also include phonological competitors.

More important, perhaps, is the fact that we did not find evidence for the proposed greater reliance of older adults on lexical frequency during target word recognition. While there 
was a significant interaction between age and lexical frequency, it occurred on the cubic time term only and was driven by a greater difference between the high- and low-frequency model fits for young adults as compared to older adults. Thus, although Revill and Spieler (2012) found stronger competition from high-frequency distractors in older adults, there is still little evidence supporting the hypothesis that older adults are more affected by the frequency of target words. Interestingly, we also found that young adults in noise (+3 dB SNR) showed similar time courses of word recognition as older adults in quiet. On one hand, this might suggest that agerelated changes in spoken word recognition are primarily impacted by age-related changes in auditory processing (given that changing the acoustic demands can produce "older adult"-like performance in young adults). However, it is important to remember that understanding speech in noise also increases cognitive demand. Thus, young adults listening to speech in noise face increases in both acoustic and cognitive challenge compared to listening in quiet, which results in a slowing of spoken word recognition similar to that seen in normal aging.

Another pattern worth mentioning in these results is that the high frequency words in noise were still recognized more quickly than low-frequency words in quiet (see Figure 4), indicating that the frequency effect is quite robust (i.e., acoustic degradation of the highfrequency words did not slow them to the level of low-frequency words). We purposely selected a noise level at which listeners would still correctly identify target words for this study, but further manipulation of SNR is needed to better delineate the relative effects of noise and word frequency on the time course of word recognition.

The fact that we did not include phonological competitors in the visual array is an important methodological and theoretical point. In real world situations, listeners have a wide array of lexical candidates to choose from: the acoustic information helps narrow down the list of candidates (in theory, up to a listener's entire lexicon) down to a single percept. Of course, in 
everyday communication, there are other syntactic and semantic constraints that help to limit the number of likely competitors. However, lexical competition still occurs in the context of a listener's internal representations. In studies that force a listener to choose from among presented options (including the current study), it is possible that the presented choices fundamentally alter the lexical competition process. That is, if a phonological competitor is included, it might introduce competition in the context of the experiment that would not be present in everyday situations. Sommers et al. (1997), for example, showed that closed-set tests of speech recognition did not reveal effects of lexical difficulty that could be observed in open-set tasks. Since our primary interest in this study was on the effect of word frequency on the time course of word recognition, we wanted to avoid any spurious competition effects introduced by including phonological competitors in the experimental array. While frequency effects without phonological competitors have been previously reported for young adults (Dahan et al., 2001), no eyetracking study with older adults, to our knowledge, has utilized competitor-less displays.

In summary, we have shown that young and older adults' spoken word recognition appears to be similarly affected by word frequency. Although we observe age differences when presenting materials in the same level of noise to both groups of listeners, adding noise to the young adults results in comparable patterns of lexical activation to the older adults in quiet. These findings are consistent with similar processes supporting spoken word recognition in young and older adults that are sensitive to both auditory and cognitive aspects of speech recognition.

\section{References}


Allopenna, P. D., Magnuson, J. S., \& Tanenhaus, M. K. (1998). Tracking the time course of spoken word recognition using eye movements: Evidence for continuous mapping models. Journal of Memory and Language, 38(4), 419-439. https://doi.org/10.1006/jmla.1997.2558

Balota, D. A., Cortese, M. J., Sergent-Marshall, S. D., Spieler, D. H., \& Yap, M. (2004). Visual word recognition of single-syllable words. Journal of Experimental Psychology. General, 133(2), 283316. https://doi.org/10.1037/0096-3445.133.2.283

Cruickshanks, K. J., Wiley, T. L., Tweed, T. S., Klein, B. E., Klein, R., Mares-Perlman, J. A., \& Nondahl, D. M. (1998). Prevalence of hearing loss in older adults in Beaver Dam, Wisconsin. The Epidemiology of Hearing Loss Study. American Journal of Epidemiology, 148(9), 879-886. https://doi.org/10.1093/oxfordjournals.aje.a009713

Dahan, D., Magnuson, J. S., \& Tanenhaus, M. K. (2001). Time course of frequency effects in spokenword recognition: evidence from eye movements. Cognitive Psychology, 42(4), 317-367. https://doi.org/10.1006/cogp.2001.0750

Goldinger, S. D., Luce, P. A., \& Pisoni, D. B. (1989). Priming Lexical Neighbors of Spoken Words: Effects of Competition and Inhibition. Journal of Memory and Language, 28(5), 501-518. https://www.ncbi.nlm.nih.gov/pubmed/24465086

Grosjean, F. (1980). Spoken word recognition processes and the gating paradigm. Perception \& Psychophysics, 28(4), 267-283. https://doi.org/10.3758/bf03204386

Homans, N. C., Metselaar, R. M., Dingemanse, J. G., van der Schroeff, M. P., Brocaar, M. P., Wieringa, M. H., Baatenburg de Jong, R. J., Hofman, A., \& Goedegebure, A. (2017). Prevalence of age-related hearing loss, including sex differences, in older adults in a large cohort study. The Laryngoscope, 127(3), 725-730. https://doi.org/10.1002/lary.26150

Howes, D. (1957). On the Relation between the Intelligibility and Frequency of Occurrence of English Words. The Journal of the Acoustical Society of America, 29(2), 296-305. https://doi.org/10.1121/1.1908862

Humes, L. E., Busey, T. A., Craig, J., \& Kewley-Port, D. (2013). Are age-related changes in cognitive function driven by age-related changes in sensory processing? Attention, Perception \& 
Psychophysics, 75(3), 508-524. https://doi.org/10.3758/s13414-012-0406-9

Luce, P. A., \& Pisoni, D. B. (1998). Recognizing spoken words: The Neighborhood Activation Model. Ear and Hearing, 19(1), 1-36. https://doi.org/10.1097/00003446-199802000-00001

Marslen-Wilson, W. D. (1987). Functional parallelism in spoken word-recognition. Cognition, 25(1-2), 71-102. https://doi.org/10.1016/0010-0277(87)90005-9

Marslen-Wilson, W. D., \& Tyler, L. K. (1980). The temporal structure of spoken language understanding. Cognition, 8(1), 1-71. https://www.ncbi.nlm.nih.gov/pubmed/7363578

Mirman, D. (2014). Growth Curve Analysis and Visualization Using R (Chapman \& Hall/CRC The R Series) (1 edition). Chapman and Hall/CRC. https://www.amazon.com/Growth-Curve-AnalysisVisualization-Chapman/dp/1466584327

Norris, D., \& McQueen, J. M. (2008). Shortlist B: a Bayesian model of continuous speech recognition. Psychological Review, 115(2), 357-395. https://doi.org/10.1037/0033-295X.115.2.357

Peelle, J. E. (2018). Listening Effort: How the Cognitive Consequences of Acoustic Challenge Are Reflected in Brain and Behavior. Ear and Hearing, 39(2), 204-214. https://doi.org/10.1097/AUD.0000000000000494

Pichora-Fuller, M. K., Kramer, S. E., Eckert, M. A., Edwards, B., Hornsby, B. W. Y., Humes, L. E., Lemke, U., Lunner, T., Matthen, M., Mackersie, C. L., Naylor, G., Phillips, N. A., Richter, M., Rudner, M., Sommers, M. S., Tremblay, K. L., \& Wingfield, A. (2016). Hearing impairment and cognitive energy: The Framework for Understanding Effortful Listening (FUEL). Ear and Hearing, 37 Suppl 1, 5S - 27S. https://doi.org/10.1097/AUD.0000000000000312

Revill, K. P., \& Spieler, D. H. (2012). The effect of lexical frequency on spoken word recognition in young and older listeners. Psychology and Aging, 27(1), 80-87. https://doi.org/10.1037/a0024113

Sommers, M. S., \& Danielson, S. M. (1999). Inhibitory processes and spoken word recognition in young and older adults: the interaction of lexical competition and semantic context. Psychology and Aging, 14(3), 458-472. https://www.ncbi.nlm.nih.gov/pubmed/10509700

Sommers, M. S., Kirk, K. I., \& Pisoni, D. B. (1997). Some considerations in evaluating spoken word recognition by normal-hearing, noise-masked normal-hearing, and cochlear implant listeners. I: The 
effects of response format. Ear and Hearing, 18(2), 89-99. https://doi.org/10.1097/00003446199704000-00001

Spieler, D. H., \& Balota, D. A. (2000). Factors influencing word naming in younger and older adults. Psychology and Aging, 15(2), 225-231. https://doi.org/10.1037/0882-7974.15.2.225

Tyler, L. K., \& Wessels, J. (1983). Quantifying contextual contributions to word-recognition processes. Perception \& Psychophysics, 34(5), 409-420. https://doi.org/10.3758/bf03203056

Wechsler, D. (2008). Wechsler adult intelligence scale--Fourth Edition (WAIS--IV). San Antonio, TX: NCS Pearson, 22, 498. https://pdfs.semanticscholar.org/2858/f906a462c4424192f80361f689bdec24c16d.pdf

Wingfield, A., Aberdeen, J. S., \& Stine, E. A. (1991). Word onset gating and linguistic context in spoken word recognition by young and elderly adults. Journal of Gerontology, 46(3), P127-P129. https://doi.org/10.1093/geronj/46.3.p127 


\section{Contributions}

Contributed to conception and design: KVE, AD, NR, BS, MS, JP

Contributed to acquisition of data: AD, NR, BS

Contributed to analysis and interpretation of data: KVE, JP

Drafted and/or revised the article: KVE, JP

Approved the submitted version for publication: KVE, AD, BS, MS, JP

\section{Acknowledgments}

We thank Kirk Ballew for assistance with data collection.

\section{Funding information}

Research reported here was supported by grant R01DC014281 from the US National Institutes of Health (Jonathan Peelle, PI; Mitch Sommers and Kristin Van Engen, Co-Investigators).

\section{Competing interests}

None of the authors have any competing interests.

\section{Supplemental Material}

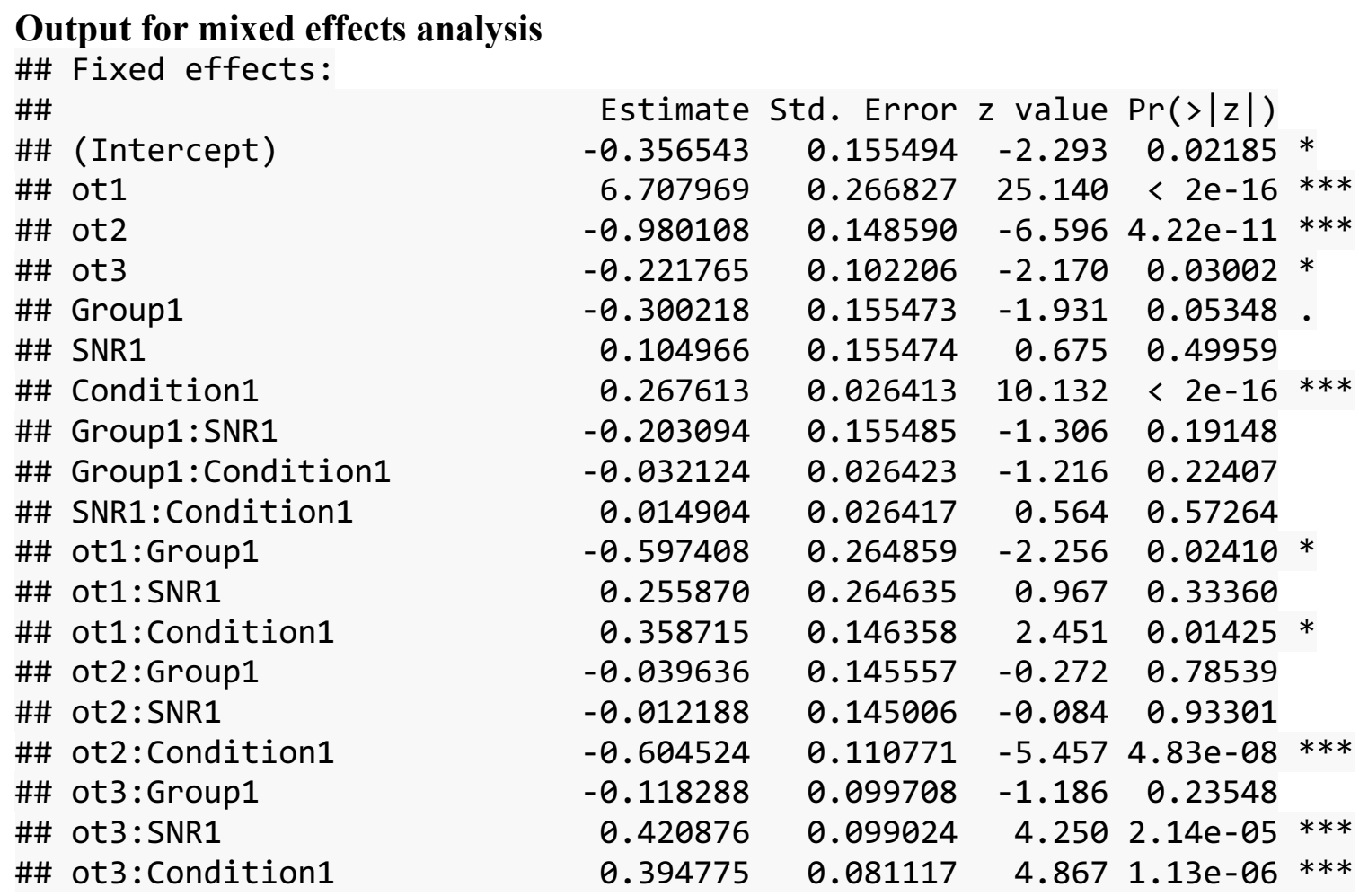




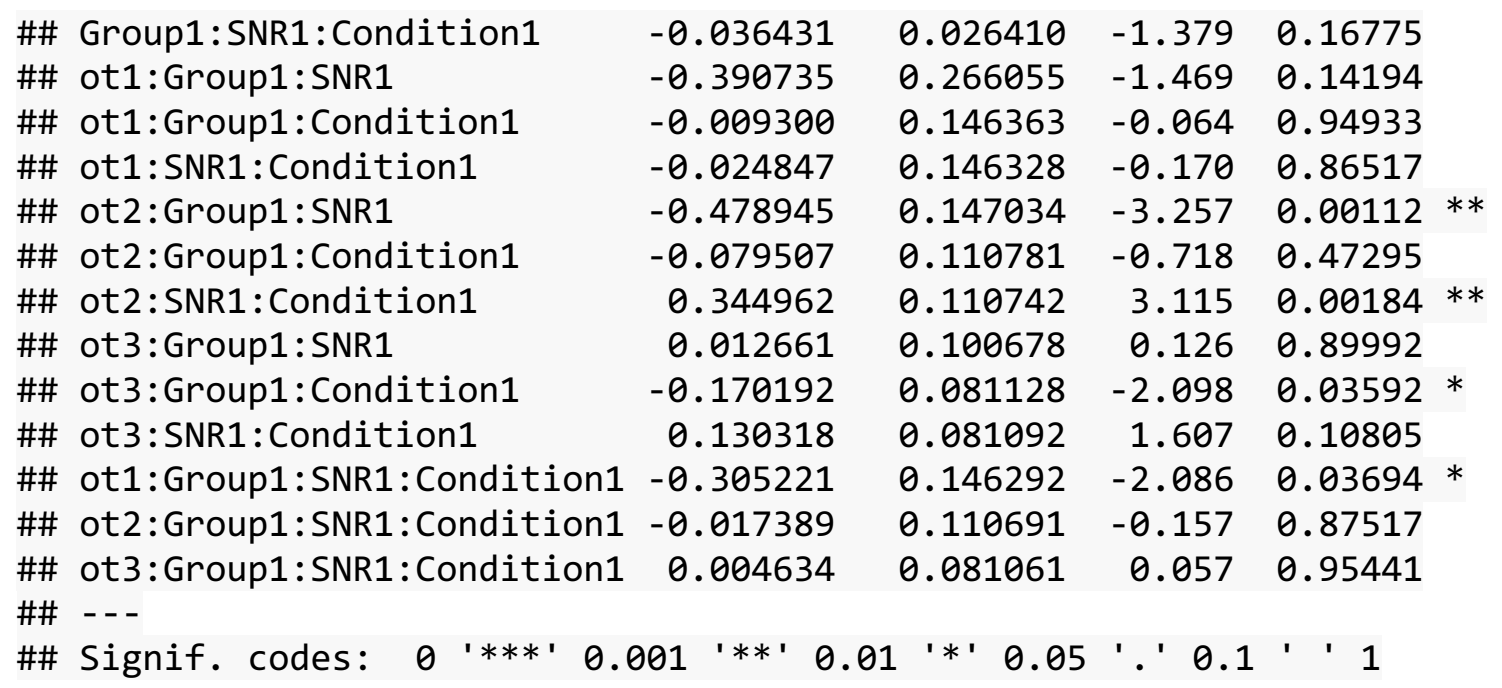

Analysis of younger adults in noise compared with older adults in quiet

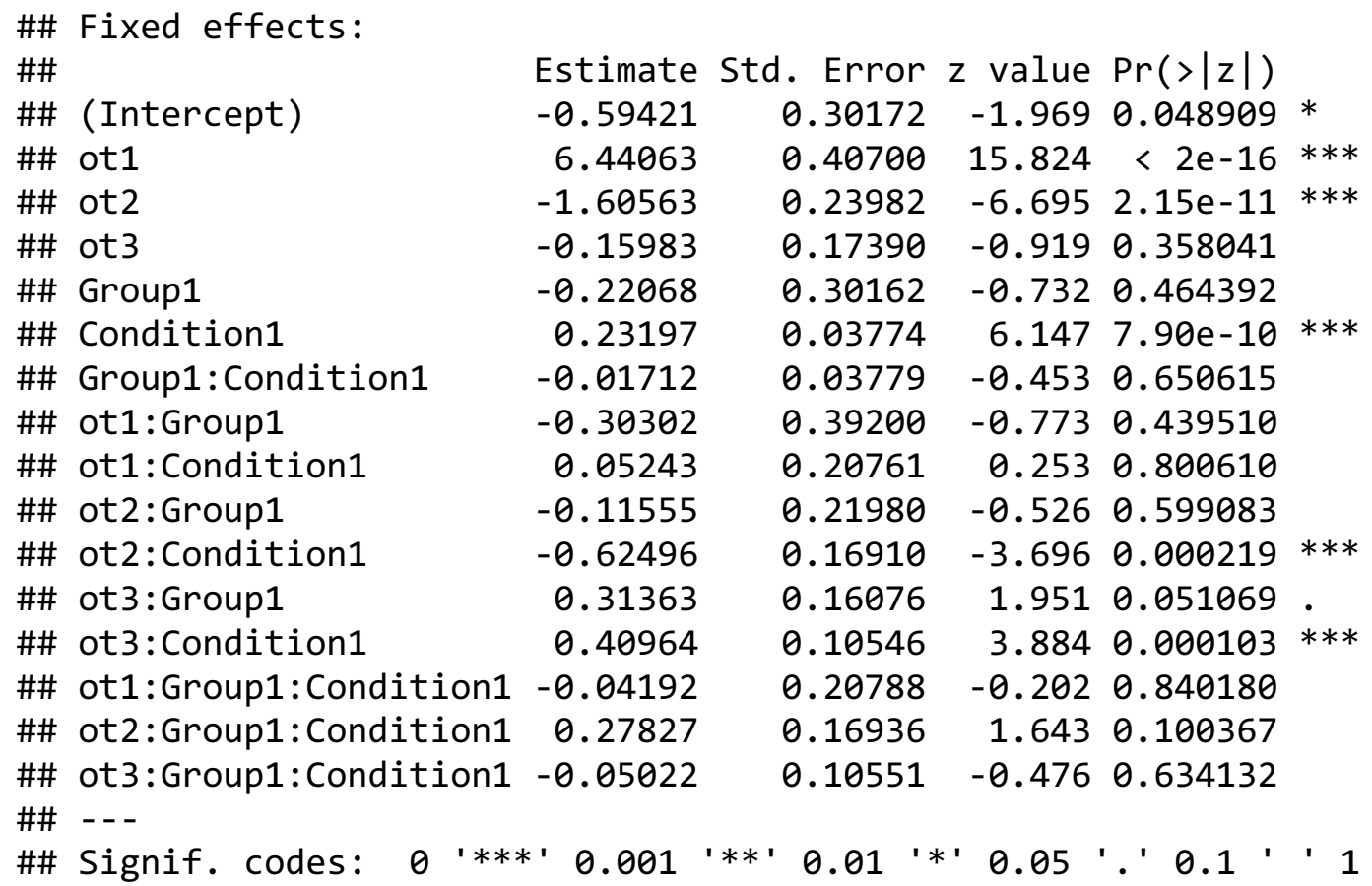

